\title{
The tuned absorptance in multilayer graphene-dielectric structures by intraband transition
}

C. H. Yang, G. X. Wang, C. Zhang, and Z. M. Ao

Citation: Journal of Applied Physics 122, 133109 (2017); doi: 10.1063/1.4991431

View online: https://doi.org/10.1063/1.4991431

View Table of Contents: http://aip.scitation.org/toc/jap/122/13

Published by the American Institute of Physics

\section{Articles you may be interested in}

Investigation of leaky and bound modes of graphene surface plasmons

Journal of Applied Physics 122, 133113 (2017); 10.1063/1.5006061

On the optical performance of composite structures of graphene and photonic crystals at infrared wavelengths Journal of Applied Physics 122, 133104 (2017); 10.1063/1.4998478

Theory of energy and power flow of plasmonic waves on single-walled carbon nanotubes Journal of Applied Physics 122, 133103 (2017); 10.1063/1.4997454

Plasmon polariton enhanced mid-infrared photodetectors based on Ge quantum dots in $\mathrm{Si}$ Journal of Applied Physics 122, 133101 (2017); 10.1063/1.4986986

A nanoscale pn junction in series with tunable Schottky barriers Journal of Applied Physics 122, 134304 (2017); 10.1063/1.4994194

Enhanced and one-way absorptance of $\mathrm{LiNiO}_{2}$ thin films in one-dimensional photonic crystals Journal of Applied Physics 122, 243104 (2017); 10.1063/1.5007818

\section{PHYSICS TODAY}

MANACER'S GUIDE WHITEPAPERS
READ NOW

PRESENTED BY

Accelerate R\&D with Multiphysics Simulation $\checkmark \subset \bigcirc M S O L$ 


\title{
The tuned absorptance in multilayer graphene-dielectric structures by intraband transition
}

\author{
C. H. Yang, ${ }^{1,2}$ G. X. Wang, ${ }^{2}$ C. Zhang, ${ }^{3}$ and Z. M. $\mathrm{Ao}^{4}$ \\ ${ }^{1}$ School of Physics and Optoelectronic Engineering, Nanjing University of Information Science and \\ Technology, Nanjing 210044, China \\ ${ }^{2}$ Center for Clean Energy Technology, School of Mathematical and Physical Sciences, University of \\ Technology Sydney, New South Wales 2007, Australia \\ ${ }^{3}$ School of Engineering Physics, University of Wollongong, Wollongong, New South Wales 2552, Australia \\ ${ }^{4}$ Institute of Environmental Health and Pollution Control, School of Environmental Science and Engineering, \\ Guangdong University of Technology, Guangzhou, China
}

(Received 20 June 2017; accepted 20 September 2017; published online 4 October 2017)

\begin{abstract}
In this work, using the transfer-matrix method, the optical transport process is investigated, with graphene inserted into multilayer dielectric structures, theoretically and numerically in the $\mathrm{THz}$ regime. When the incident frequency is lower than the graphene Fermi energy, the optical intraband transitions provide the main contribution to the graphene surface current. The absorptance can be enhanced to about $50 \%$ with only one graphene/dielectric layer in air. When increasing the number of dielectric layers coated with graphene, the absorption increases. Periodic absorption peaks are observed in multilayer structures. The positions of the absorption peaks as a function of the frequency and the incident angle are in accordance with the positions of the abrupt change in the reflection coefficient phase and of the imaginary solution of the Bloch wavevector in expanding periodic structures using Bloch theorem. Published by AIP Publishing.
\end{abstract}

https://doi.org/10.1063/1.4991431

\section{INTRODUCTION}

Two-dimensional (2D) graphene materials fabricated experimentally in 2004 by Geim et al., ${ }^{1}$ have been arousing tremendous interest in condensed matter physics ${ }^{2-6}$ due to their superior electronic and optical properties, such as the unique quantum Hall effect, ${ }^{1}$ high mobility, ${ }^{7}$ Klein tunnelling, ${ }^{8}$ dynamic graphene conductivity, ${ }^{9,10}$ and so on. The photonic and optoelectronic applications depend on the high optical absorption of atomic graphene with interaction between the propagating photons and the graphene. The tunable optical reflectivity, transmissivity, and absorptivity have been investigated in many graphene-based systems, such as graphene nanodisc arrays, ${ }^{11,12}$ graphene nanoribbons, ${ }^{13}$ alternating dielectric media with graphene layers sandwiched between them, ${ }^{14-16}$ graphene-based Salisbury screens, ${ }^{17}$ stacked graphene pairs or graphene photonic crystals, etc. ${ }^{18-22}$ In a multilayer structural system, the optical transport properties and transmission are dependent on the number of dielectric and graphene layers, the dielectric space thickness, the incident angle, the incident light wavelength (or frequency) which is located in different graphene intraand/or inter-band optical transition regions, the surface plasmon-polaritons, and the transverse electric/transverse magnetic (TE/TM) modes. So many factors can be used to tune the optical properties of graphene-based dielectric structure systems which can meet the requirements of optoelectronic applications. As an example, Sensale-Rodriguez et al $^{16,19}$ designed structures on " $\mathrm{SiO}_{2} / \mathrm{Si}$ " dielectric substrates coated with graphene. It can be a back electrode plane which acts as a reflector, or a ring-shaped gate that can transmit light through the system. The optical transmittance can be experimentally modulated by $15 \%$ in the ring-shaped bottom gate at about $570 \mathrm{GHz} .{ }^{19}$ When using the planar back electrode as a reflector, the reflectance can be controlled by the optical thickness of the substrate. ${ }^{16}$ The influence of a nonzero mass-gap on the reflectivity properties has also been investigated at both zero and nonzero temperatures in graphene. $^{23}$

When graphene is embedded between two dielectric layers, the graphene layer can be treated as a homogeneous medium with an effective thickness ${ }^{20,24}$ or as a boundary with the interface conductivity. ${ }^{14}$ At the interface between different dielectric layers, the electric and magnetic fields satisfy the boundary conditions. The amplitude reflection and transmission coefficients in the presence of graphene at the interface have been obtained by the modified Fresnel formula $^{25}$ and a quantum field theoretical description. ${ }^{26}$ Michele Merano investigated the optical transmission properties in a two-layer dielectric structure with graphene deposited between them. ${ }^{27}$ In his work, graphene was treated by two methods, as a homogeneous slab with an effective dielectric function or as surface graphene conductivity included in the modified Fresnel formulas. The results showed that the Fresnel-based model is superior to the other one.

There are intra- and inter-band optical transitions among or between the valence band and the conduction band which contribute to graphene's optical conductivity. ${ }^{9,10}$ From the infrared to visible wavelength regime, the optical conductivity of monolayer graphene is almost a constant of $e^{2} /(4 \hbar)$ from the inter-band optical transition process. In the terahertz region, the Drude optical conductivity $(4-100) e^{2} /(4 \hbar)$ was observed accompanied by the intra-band transitions. ${ }^{28,29}$ The 
conductivity value from intra-band transition is much larger than that from the inter-band transition. Recently, using the polarization tensor, the numerical computations of conductivity of pure graphene ${ }^{30}$ and of gapped graphene ${ }^{31}$ were performed in the framework of quantum electrodynamics at non-zero and zero temperatures. This conductivity can be modulated by the voltage, ${ }^{32-34}$ optical methods, ${ }^{35-37}$ electron-phonon coupling, ${ }^{38}$ temperature, disorder, and so on. Various structures with graphene layers inserted were designed and used to increase the optical absorption, mostly using the graphene inter-band optical transition conductivity. The investigation done by Sensale-Rodriguez et al. showed that the transmittance and the reflectance can be modulated by graphene conductivity from the intra-band optical transition. $^{16,19}$

Motivated by these studies, in this paper, we investigate the optical absorption properties in graphene-based multilayer dielectric structures. We focus on the graphene absorptance in the graphene intra-band transition region. The positions of the absorption peaks can be understood from the phase of the reflection coefficient and the energy band structure for the extended periodic dielectric structure.

\section{THEORETICAL APPROACHES}

In this paper, we consider a composite multilayer material alternating stacked homogeneous graphene sheets and dielectric layers, each with a thickness of $d$. The refractive index of silicon can be described by the Herzberger-type dispersion formula as $n(\lambda)=A+B L+C L^{2}+D \lambda^{2}+E \lambda^{4}$, where $L=1 /\left(\lambda^{2}-0.028\right)$ with the wavelength $\lambda$ in micrometers. From 1.12 to $588 \mu \mathrm{m}$, the coefficients are $A=3.41906, B=0.123172, C=0.0265456, D=-2.66511$ $\times 10^{-8}$, and $E=5.45852 \times 10^{-14}$. A single atomic layer graphene is treated as a conducting surface layer between different dielectric media. The TE (TM) mode is defined as the component of the electric (magnetic) field parallel to the interface. The electric and magnetic fields in an arbitrary layer $j$ for the TE wave along the propagating $z$-direction can be expressed as

$$
\left(\begin{array}{c}
E_{j}(z) \\
H_{j}(z)
\end{array}\right)=\left(\begin{array}{cc}
e^{i k_{z, j} z} & e^{-i k_{z, j} z} \\
-\eta_{j}^{T E} e^{i k_{z, j} z} & \eta_{j}^{T E} e^{-i k_{z, j} z}
\end{array}\right)\left(\begin{array}{l}
a_{j} \\
b_{j}
\end{array}\right),
$$

where $\eta_{j}^{T E}=k_{z, j} /\left(\omega \mu_{i}\right)$ and $k_{z}$ is the wavevector perpendicular to the 2D plane. For the TM wave, the electromagnetic field is given by

$$
\left(\begin{array}{c}
H_{j}(z) \\
E_{j}(z)
\end{array}\right)=\left(\begin{array}{cc}
e^{i k_{z, j} z} & e^{-i k_{z_{j}} z} \\
\eta_{j}^{T M} e^{i k_{z, j} z} & -\eta_{j}^{T M} e^{-i k_{z, j} z}
\end{array}\right)\left(\begin{array}{c}
a_{j} \\
b_{j}
\end{array}\right),
$$

where $\eta_{j}^{T M}=k_{z, j} /\left(\omega \varepsilon_{i}\right) . a_{j}$ and $b_{j}$ are the amplitude coefficients of the forward and backward propagating wave. In the first layer, $a_{i}=1$ was used. $b_{i}=r$ refers to the reflection coefficient of the electric (magnetic) field amplitude for the TE (TM) mode. There is no backward propagating field in the last layer. $a_{f}=t$ is the transmission coefficient and $b_{f}=0$. The subscripts $i$ and $f$ indicate the initial and final layers, respectively.
By taking into account the surface electric current on the boundary conditions for the electric and magnetic fields, rigorous transfer matrices for the TE polarization in the $j$ th layer are given as

$$
M_{j}^{T E}=\left(\begin{array}{cc}
\cos \left(k_{z, j} d_{j}\right) & \left(i / \eta_{j}^{T E}\right) \sin \left(k_{z, j} d_{j}\right) \\
i \eta_{j}^{T E} \sin \left(k_{z, j} d_{j}\right)+\alpha & \cos \left(k_{z, j} d_{j}\right)+\beta
\end{array}\right),
$$

where $\alpha=-\sigma_{j} \cos \left(k_{z, j} d_{j}\right), \beta=-\left(i \sigma_{j} / \eta_{j}^{T E}\right) \sin \left(k_{z, j} d_{j}\right), \sigma_{j}$ is the graphene conductivity and $d_{j}$ is the thickness of the $j$ th layer, and

$$
M_{j}^{T M}=\left(\begin{array}{cc}
\cos \left(k_{z, j} d_{j}\right)+\gamma & -\left(i / \eta_{j}^{T M}\right) \sin \left(k_{z, j} d_{j}\right)+\zeta \\
-i \eta_{j}^{T M} \sin \left(k_{z, j} d_{j}\right) & \cos \left(k_{z, j} d_{j}\right)
\end{array}\right)
$$

for the TM polarization. $\gamma=-\left(i \sigma_{j} \eta_{j}^{T M}\right) \sin \left(k_{z, j} d_{j}\right), \quad \zeta=\sigma_{j}$ $\cos \left(k_{z, j} d_{j}\right)$.

Using the transfer matrix, the relationship of the electromagnetic field with the first layer and the last layer for the TE and TM modes is given as

$$
X_{T E / T M}\left(\begin{array}{c}
1 \\
r
\end{array}\right)=\prod_{j=1}^{n} M_{j}^{T E / T M} Y_{T E / T M}\left(\begin{array}{c}
t \\
0
\end{array}\right) .
$$

Here,

$$
\begin{aligned}
& X_{T E}=\left(\begin{array}{cc}
1 & 1 \\
-\eta_{0}^{T E} & \eta_{0}^{T E}
\end{array}\right), \\
& Y_{T E}=\left(\begin{array}{cc}
e^{i k_{z, 0} d_{T}} & e^{-i k_{z, 0} d_{T}} \\
-\left(\eta_{0}^{T E}+\sigma_{t}\right) e^{i k_{z, 0} d_{T}} & \left(\eta_{0}^{T E}-\sigma_{t}\right) e^{-i k_{z, 0} d_{T}}
\end{array}\right), \\
& X_{T M}=\left(\begin{array}{cc}
1 & 1 \\
\eta_{0}^{T M} & -\eta_{0}^{T M}
\end{array}\right), \\
& Y_{T M}=\left(\begin{array}{cc}
\left(1+\eta_{0}^{T M} \sigma_{t}\right) e^{i k_{z, 0} d_{T}} & \left(1-\eta_{0}^{T M} \sigma_{t}\right) e^{-i k_{z, 0} d_{T}} \\
\eta_{0}^{T M} e^{i k_{z, 0} d_{T}} & -\eta_{0}^{T M} e^{-i k_{z, 0} d_{T}}
\end{array}\right)
\end{aligned}
$$

are for the TE and TM modes, respectively. The subscript " 0 " represents the parameter values in the vacuum with the $i$ and $f$ layers being air. $d_{T}$ is the total distance for one period. $\sigma_{t}$ is the graphene conductivity at the last interface. Therefore, the reflection $(r)$ and transmission $(t)$ coefficients can be obtained using Eq. (5). Then, the reflectance $|r|^{2}$, the transmittance $|t|^{2}$, and the absorptance of graphene ( $\left.A=1-|r|^{2}-|t|^{2}\right)$ can be readily obtained.

If the multilayer structures are extended to infinite periodic structures, based on Bloch theorem and the boundary condition, the dispersion relation for the TE/TM waves can be obtained as

$$
\cos \left(\beta d_{T}\right)=\frac{1}{2} \operatorname{Tr}\left(M_{1}^{T E / T M} M_{2}^{T E / T M} \ldots\right),
$$

where $\beta$ is the vertical component of the Bloch wavevector. Because of the graphene surface conductivity, this wavevector is imaginary, corresponding to the optical absorption. 


\section{RESULTS AND DISCUSSION}

In this section, using the transfer matrix formula, the graphene absorptance, the related reflection coefficient and the energy band spectrum in the periodic structures have been investigated in detail. Because the graphene monolayer material is inserted between two dielectric layers, the optical transport properties can be tuned by the gate voltage (or graphene Fermi energy) for the occurrence of the graphene surface conductivity. ${ }^{9,10}$ In the present paper, we focus on the influence on the absorptance of the graphene intra-band transition conductivity around the $\mathrm{THz}$ frequency regime. Given that the $\mathrm{SiO}_{2}$ thickness of $\sim 100 \mathrm{~nm}$ is much smaller than the $\mathrm{THz}$ wavelength with a wavelength of hundreds of $\mu \mathrm{m}$, the optical path difference when light is transmitting through this layer can be neglected. The temperature of $200 \mathrm{~K}$ and the broadening width of $0.1 \mathrm{meV}$ were used when calculating the graphene conductivity.

Figure 1 illustrates the dependence of the graphene absorptance on the incident frequency and the graphene Fermi energy from $100 \mathrm{meV}$ to $400 \mathrm{meV}$ at normal incidence in the "air-(G-Si)-air" structure. G indicates the graphene layer. When the optical energy's frequency is at hundreds of $\mathrm{GHz}$, the intra-band optical transitions dominate the contribution to the graphene conductivity. In this frequency region, the graphene surface conductivity increases with the Fermi energy from 100 to $400 \mathrm{meV}$ and decreases with increasing optical energy. The thickness of the dielectric layer Si was set to $480 \mu \mathrm{m} .{ }^{19}$ Absorption peaks are observed at frequencies of 548 and $640 \mathrm{GHz}$. For a small Fermi energy $(100 \mathrm{meV})$, the maximum absorptance is about 5.3\% . As $E_{F}$ increases, as expected, graphene absorptance increases with increasing graphene conductivity. Because of the Si space layer introduced, the absorption oscillates with the optical frequency, which is the result of the interference phenomenon, and the transmittance varies obviously around the absorption peaks. In contrast, when a graphene monolayer is

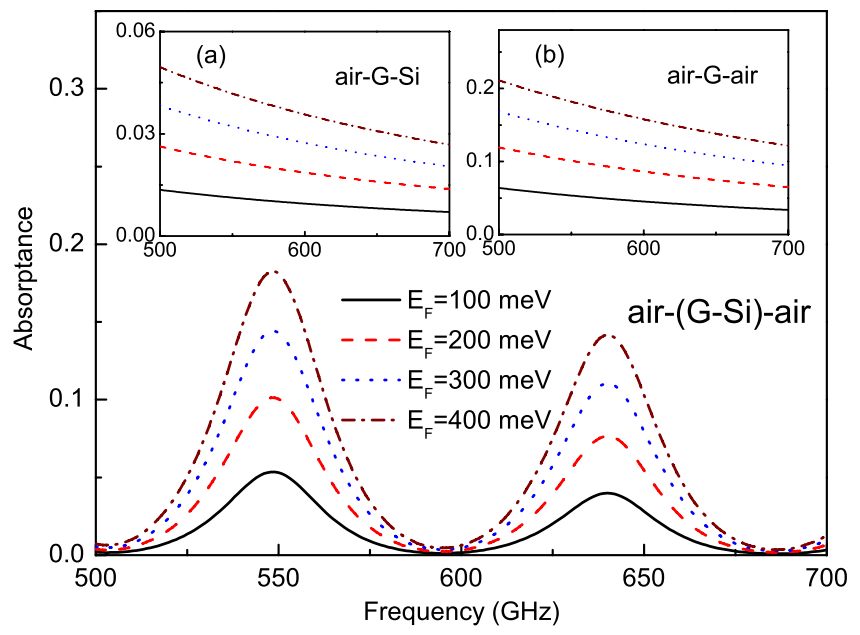

FIG. 1. The absorptance of graphene as a function of the incident optical frequency in the "air-(G-Si)-air" structure at normal incidence for various graphene Fermi energies. The black solid, red dashed, blue dotted, and brown dash-dotted lines are for $E_{F}=100,200,300$, and $400 \mathrm{meV}$, respectively. The thickness of the Si substrate is $480 \mu \mathrm{m}$. The insets (a) and (b) are the absorptances of graphene sandwiched between two dielectric layers. G indicates the graphene layer.

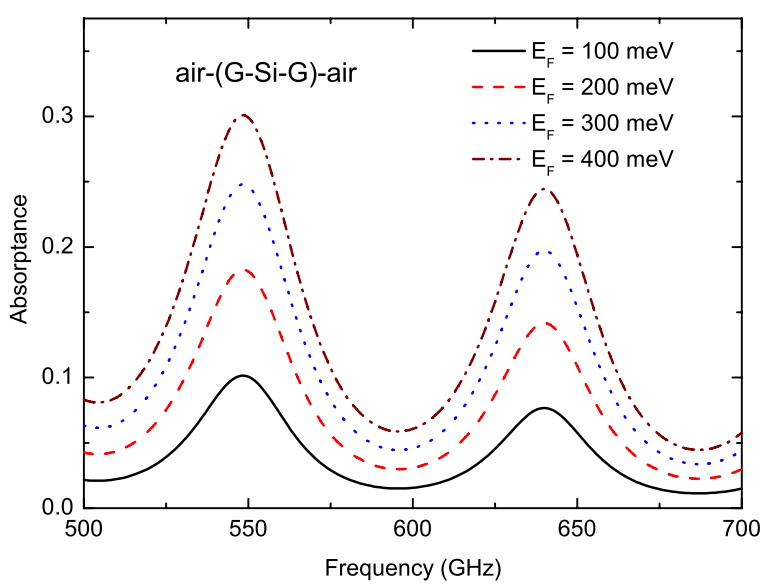

FIG. 2. Results of the "air-(G-Si-G)-air" structure, similar to Fig. 1.

only inserted between two dielectric layers, the optical absorptance varies monotonically and is proportional to the graphene conductivity, as can be seen from the insets in Fig. 1.

In order to obtain strong absorption, the structure can be extended to contain more layers of graphene and dielectric media to become a multilayer or superlattic structures. For example, in Fig. 2, another graphene layer was coated on the other side of the dielectric layer. Because of more chances of light transmitting through graphene, its absorption is expected to increase which can be seen in Fig. 2, while the positions of the strong absorption peaks are unchanging. Another feature is that the absorptance between these two absorption peaks increases when two graphene layers adhered on both sides of the dielectric layer, and the reflectance is much influenced in this frequency region. In order to investigate the angular dependence for the above structure, the graphene absorptance as a function of the incident frequency at normal and oblique incidences for the TE and TM modes is shown in Fig. 3. Two absorption peaks for two kinds of polarizations are observed from 500 to $700 \mathrm{GHz}$.

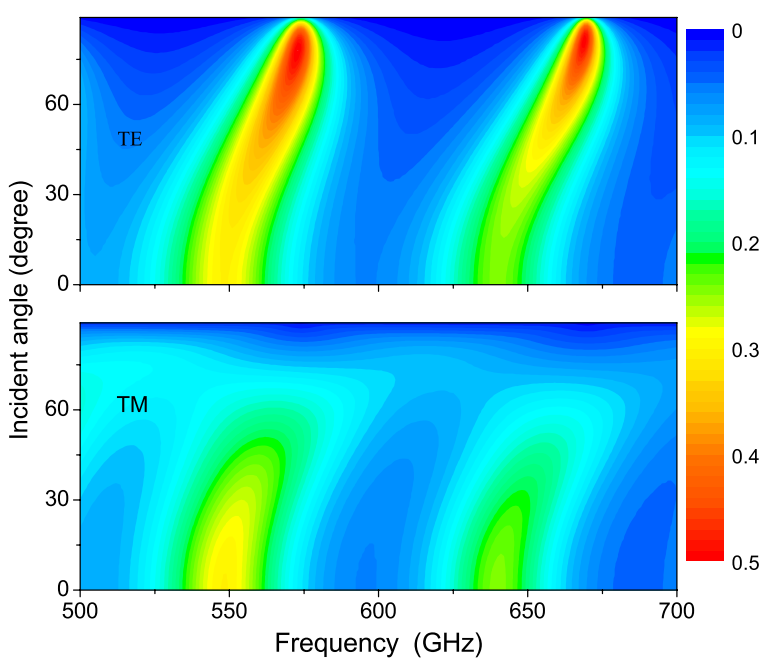

FIG. 3. The absorptance of graphene as a function of the incident optical frequency and the incident angle for the TE and TM modes in the "air-(G-Si-G)air" structure using a Si space layer with a thickness of $480 \mu \mathrm{m}$. The Fermi energy $E_{F}=400 \mathrm{meV}$. The color bar represents the value of absorptance. 
When light transmits through the dielectric structure, it should satisfy the resonance condition $2 n k d \cos \theta=m 2 \pi$, where $n, k, d$, and $\theta$ are the dielectric refractive index, the wavevector, the width, and the optical propagating angle in the spacer layer, and $m$ is an integer. Therefore, $k \cos \theta \propto m$ leads to the absorption peaks moving towards higher energy with increasing incidence angle. The dependence of absorptance on the incident angle exhibits differently for the two polarized modes. For the TE mode, the absorption becomes more and more strong with increasing incidence angle. The maximum absorptance increases from about $30 \%$ at normal incidence to about $50 \%$ at nearly grazing incidence. But for the TM mode, the strong absorption points are mainly located at small incident angles. At around $70^{\circ}$ incidence angle, the absorptance spectrum is more complicated. This different relationship for the TE and TM waves can be understood in the following discussion.

Figures 4 and 5 refer to the (G-Si) $)_{2}$ structure with one more (G-Si) layer added compared to the former case. A constant width of $480 \mu \mathrm{m}$ for each dielectric layer is used in Fig. 4, and periodic absorption peaks are observed. In general, the absorption is strengthened. With one more (G-Si) layer added, the frequency interval between the adjacent absorption peaks becomes $1 / 2$ of its original value, which is the result of interference and phase matching provided by the additional dielectric layer. It is also noted that there are several strong absorption points at nearly grazing incidence angles for the TM mode. In Fig. 5, we fix the total width of the dielectric layer, which means that a width of $480 / \mathrm{N} \mu \mathrm{m}$ for each dielectric layer is used, where $\mathrm{N}$ is the total number of dielectric layers. In Fig. 5, N=2 and 4 for (G-Si) $)_{N}$ layers. The numerical results show that with the increase of the dielectric layers together with the graphene layers an obviously stronger absorption was observed for both the TE and TM cases.

To understand the physical mechanism of enhanced absorption in multilayer structures with graphene inserted, Figs. 6 and 7 depict the phase of the reflection coefficient

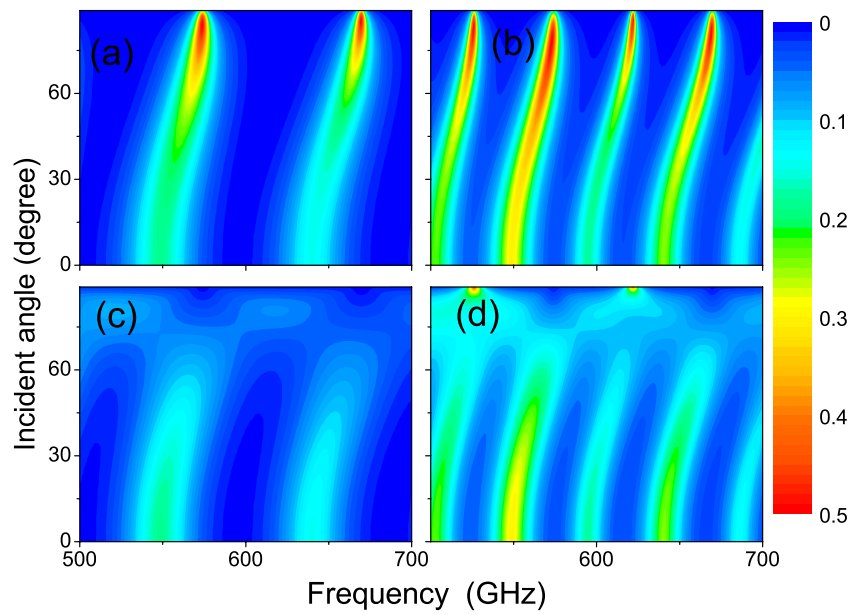

FIG. 4. The absorptance of graphene as a function of the incident optical frequency and the incident angle for the TE (a) and (b) and the TM (c) and (d) modes in an "air-(G-Si)-air" structure (a) and (c), and in an "air-(G-Si) $)_{2}$-air" structure (b) and (d). The thickness of each Si layer is $480 \mu \mathrm{m}$. The Fermi energy $E_{F}=400 \mathrm{meV}$. The color bar represents the value of absorptance.

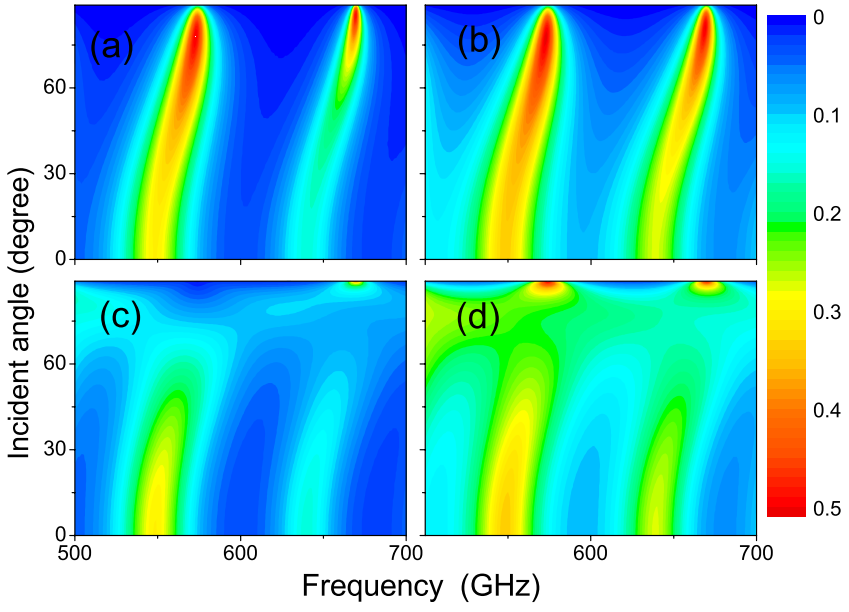

FIG. 5. (G-Si) $)_{2,4}$ structures in a similar case as in Fig. 4, but with a fixed total dielectric thickness of $480 \mu \mathrm{m}$ which indicates the thickness of each (G-Si) being $480 / \mathrm{N} \mu \mathrm{m} . \mathrm{N}$ is the number of layers in $(\mathrm{G}-\mathrm{Si})_{N}$. The Fermi energy $E_{F}=400 \mathrm{meV}$. The color bar represents the value of absorptance.

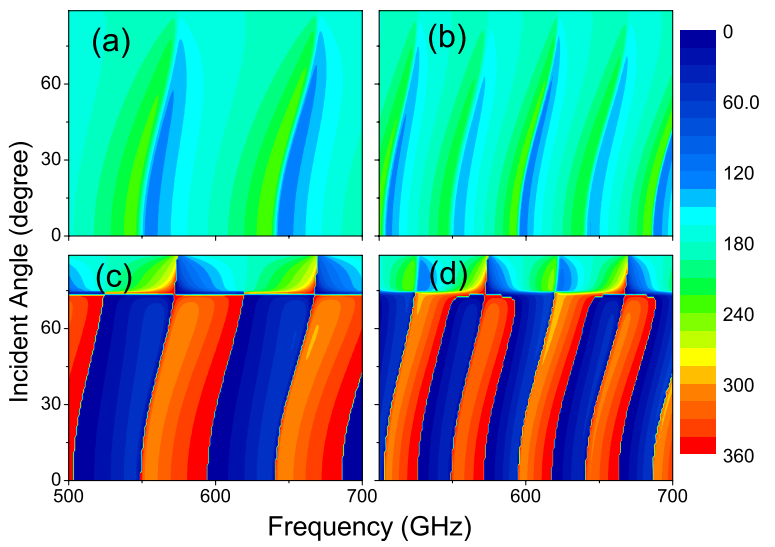

FIG. 6. The phase of the reflection coefficient as a function of the incident optical frequency and the incident angle for the TE (a) and (b) and the TM (c) and (d) modes. The structures and the parameters are the same as in Fig. 4. The color bar represents the value of phase change in the amplitude reflection coefficient $\left(0^{\circ}-360^{\circ}\right)$.

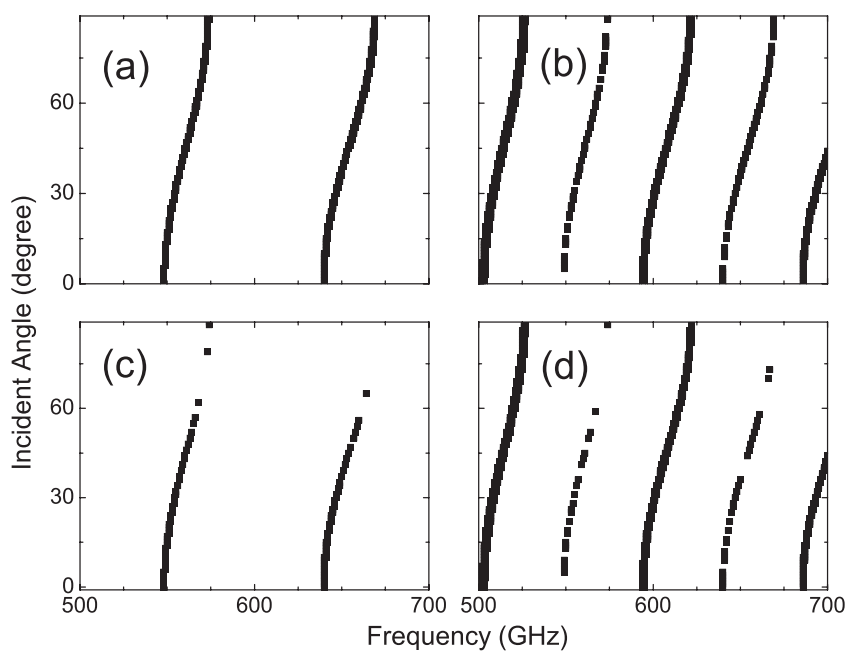

FIG. 7. The modulus of the right hand side of Eq. (6) greater than 1 as a function of the incident optical frequency and the incident angle for the TE (a) and (b) and the TM (c) and (d) modes. The structures and the parameters are the same as in Fig. 4. 
using Eq. (5) and the energy band information obtained by Bloch theorem versus the incident angle and the optical frequency. The most intriguing phenomena are that the phase of the amplitude reflection coefficient of the electric and magnetic fields exhibits sudden changes for both the TE and TM modes. Moreover, the positions of the phase sudden change are coincident with the strongest absorption lines as a function of the frequency and the incident angle. When increasing the dielectric layer, the number of abrupt changes in the reflection phase increases. The TM case is complicated for the graphene absorptance spectrum and can be understood from the phase map of the reflection coefficient [Figs. 6(c) and 6(d)]. Above or below about $73.7^{\circ}$ of the incident angle (which relates to the Brewster angle at the air and dielectric interface), the phase change was observed in an opposite way. Another way to understand the absorption peaks is the energy band structure in periodic dielectric structures. In the present paper, the layer number is finite, and the optical transmission is from air to the Si dielectric material. The photon states can be employed to analyze the transport properties in a corresponding periodic structure. Therefore, we extend air-(G-Si) to an infinite periodic structure. Based on Bloch theorem and the electromagnetic field boundary condition, the dispersion relation for the photon states in the periodic structures can be obtained using Eq. (6). Without the graphene surface conductivity, the modulus of the right side of Eq. (6) greater than 1 indicates energy band gaps. However, in the presence of graphene, the Bloch wavevector is complex and the imaginary part of the Bloch wavevector relates to the optical absorption. In order to obtain the main details of optical propagation in the dielectric layer, the width of the air layer is assumed to be $1 \mu \mathrm{m}$. Figure 7 shows the results for $\left|\operatorname{Tr}\left(M_{1}^{T E / T M} M_{2}^{T E / T M} \ldots\right) / 2\right|>1$ which match with the absorption peaks totally.

In summary, using the transfer matrix approach and taking into account the electric and magnetic boundary continuous condition, we have theoretically studied the optical transport process in multilayer dielectric structures with surface graphene conductivity included both for the TE and TM modes. When only one layer of graphene material is sandwiched between two dielectric layers, the dependence of graphene absorptance on the optical incident frequency at different graphene Fermi energies is monotonic. In the presence of the multilayer system, a periodic absorptance has been observed as the result of interference phenomena. The maximum absorption peaks occur at destruction interference for the reflective coefficient. From normal incidence to grazing incidence, the absorption peaks for both the TE and TM modes shift towards a high frequency to satisfy the interference condition. The graphene absorptance for the TE mode increases with increasing incident angle. But, for the TM mode, the absorption becomes weak, and around the Brewster angle, the absorptance is complicated. In order to obtain strong absorption, more (G-dielectric) layers can be stacked into multilayer structures. More chances for optical beams to go through the graphene layers together with the interference contribution lead to the graphene absorptance increase. The absorption peaks can be analyzed and interpreted from the phase of the reflection coefficient, and the energy band spectrum for the corresponding periodic structures. The positions of the absorption peaks are in accordance with the positions of the abrupt change in the reflective coefficient phase and the imaginary solution of the Bloch wavevector for the periodic dielectric patterns which give rise to strong absorption. In addition, graphene optical conductivity can be tuned by optical and/or electrical methods, which can enhance the graphene absorption property in graphene-based structures.

\section{ACKNOWLEDGMENTS}

This work was supported by the China Scholarship Council (CSC), the Natural Science Foundation of Jiangsu Province (BK20131428), and the National Science Foundation of China (11547030, 21607029, and 21777033).

${ }^{1}$ K. S. Novoselov, A. K. Geim, S. V. Morozov, D. Jiang, M. I. Katsnelson, I. V. Grigorieva, S. V. Dubonos, and A. A. Firsov, Nature (London) 438, 197 (2005) ; Y. Zhang, Y.-W. Tan, H. L. Stormer, and P. Kim, ibid. 438, 201 (2005).

${ }^{2}$ A. H. Castro Neto, F. Guinea, N. M. R. Peres, K. S. Novoselov, and A. K. Geim, Rev. Mod. Phys. 81, 109 (2009).

${ }^{3}$ S. Das Sarma, S. Adam, E. H. Hwang, and E. Rossi, Rev. Mod. Phys. 83, 407 (2011).

${ }^{4}$ V. N. Kotov, B. Uchoa, V. M. Pereira, F. Guinea, and A. H. Castro Neto, Rev. Mod. Phys. 84, 1067 (2012).

${ }^{5}$ R. Roldán, M. O. Goerbig, and J.-N. Fuchs, Semicond. Sci. Technol. 25, 034005 (2010).

${ }^{6}$ M. Orlita and M. Potemski, Semicond. Sci. Technol. 25, 063001 (2010).

${ }^{7}$ S. V. Morozov, K. S. Novoselov, M. I. Katsnelson, F. Schedin, D. C. Elias, J. A. Jaszczak, and A. K. Geim, Phys. Rev. Lett. 100, 016602 (2008).

${ }^{8}$ M. I. Katsnelson, K. S. Novoselov, and A. K. Geim, Nat. Phys. 2, 620-625 (2006).

${ }^{9}$ L. A. Falkovsky and S. S. Pershoguba, Phys. Rev. B 76, 153410 (2007).

${ }^{10}$ G. W. Hansona, J. Appl. Phys. 103, 064302 (2008).

${ }^{11}$ S. Thongrattanasiri, F. H. L. Koppens, and F. Javier García de Abajo, Phys. Rev. Lett. 108, 047401 (2012).

${ }^{12}$ Z. Fang, Y. Wang, A. E. Schlather, Z. Liu, P. M. Ajayan, F. Javier García de Abajo, P. Nordlander, X. Zhu, and N. J. Halas, Nano Lett. 14, 299 (2014).

${ }^{13}$ H. Yan, T. Low, W. Zhu, Y. Wu, M. Freitag, X. Li, F. Guinea, P. Avouris, and F. Xia, Nat. Photonics 7, 394 (2013).

${ }^{14}$ Y. V. Bludov, M. I. Vasilevskiy, and N. M. R. Peres, Europhys. Lett. 92, 68001 (2010).

${ }^{15}$ T. Otsuji, T. Watanabe, S. A. Boubanga Tombet, A. Satou, W. M. Knap, V. V. Popov, M. Ryzhii, and V. Ryzhii, IEEE Trans. Terahertz Sci. Technol. 3, 63 (2013).

${ }^{16}$ B. Sensale-Rodriguez, R. Yan, S. Rafique, M. Zhu, W. Li, X. Liang, D. Gundlach, V. Protasenko, M. M. Kelly, D. Jena, L. Liu, and H. G. Xing, Nano Lett. 12, 4518 (2012).

${ }^{17}$ X. Ying, Y. Pu, Z. Li, Z. Liu, and Y. Jiang, J. Opt. 44, 59 (2015).

${ }^{18}$ L. Zhang, G. Wang, X. Hana, and Y. Zhao, Optik 127, 2030 (2016).

${ }^{19}$ B. Sensale-Rodriguez, R. Yan, M. M. Kelly, T. Fang, K. Tahy, W. S. Hwang, D. Jena, L. Liu, and H. G. Xing, Nat. Commun. 3, 780 (2012).

${ }^{20}$ J. T. Liu, N. H. Liu, J. Li, X. J. Li, and J. H. Huang, Appl. Phys. Lett. 101, 052104 (2012).

${ }^{21}$ S. R. Entezar, Z. Saleki, and A. Madani, Physica B 478, 122 (2015).

${ }^{22}$ A. Madani and S. R. Entezar, Physica B 431, 1 (2013).

${ }^{23}$ G. L. Klimchitskaya and V. M. Mostepanenko, Phys. Rev. A 93, 052106 (2016).

${ }^{24}$ P. Blake, E. W. Hill, A. H. Castro Neto, K. S. Novoselov, D. Jiang, R. Yang, T. J. Booth, and A. K. Geim, Appl. Phys. Lett. 91, 063124 (2007).

${ }^{25}$ B. E. Sernelius, Phys. Rev. B 85, 195427 (2012).

${ }^{26}$ M. Bordag, G. L. Klimchitskaya, V. M. Mostepanenko, and V. M. Petrov, Phys. Rev. D 91, 045037 (2015).

${ }^{27}$ M. Merano, Phys. Rev. A 93, 013832 (2016). 
${ }^{28}$ Z. Q. Li, E. A. Henriksen, Z. Jiang, Z. Hao, M. C. Martin, P. Kim, H. L. Stormer, and D. N. Basov, Nat. Phys. 4, 532 (2008).

${ }^{29}$ A. B. Kuzmenko, E. van Heumen, F. Carbone, and D. van der Marel, Phys. Rev. Lett. 100, 117401 (2008).

${ }^{30}$ G. L. Klimchitskaya and V. M. Mostepanenko, Phys. Rev. B 93, 245419 (2016).

${ }^{31}$ G. L. Klimchitskaya and V. M. Mostepanenko, Phys. Rev. B 94, 195405 (2016).

${ }^{32}$ M. Ryzhii and V. Ryzhii, Jpn. J. Appl. Phys. 46, L151 (2007).

${ }^{33}$ X. G. Xu, S. Sultan, C. Zhang, and J. C. Cao, Appl. Phys. Lett. 97, 011907 (2010).
${ }^{34}$ D. Svintsov, V. Vyurkov, V. Ryzhii, and T. Otsuji, J. Appl. Phys. 113(5), 053701 (2013).

${ }^{35}$ V. Ryzhii, M. Ryzhii, A. Satou, T. Otsuji, A. A. Dubinov, and V. Y. Aleshkin, J. Appl. Phys. 106, 084507 (2009).

${ }^{36}$ V. Ryzhii, M. Ryzhii, and T. Otsuji, J. Appl. Phys. 101, 083114 (2007).

${ }^{37}$ H. Yang, X. Feng, Q. Wang, H. Huang, W. Chen, A. T. S. Wee, and W. Ji, Nano Lett. 11(7), 2622 (2011).

${ }^{38}$ E. Pomarico, M. Mitrano, H. Bromberger, M. A. Sentef, A. AlTemimy, C. Coletti, A. Stöhr, S. Link, U. Starke, C. Cacho, R. Chapman, E. Springate, A. Cavalleri, and I. Gierz, Phys. Rev. B 95, 024304 (2017). 